\title{
Grafen Temelli İletken Polimer-Nanokompozit Elektrodunun Sentezi, Elektrokimyasal ve Optik Özelliklerinin İncelenmesi
}

\author{
Rukiye AYRANCI \\ Pamukkale Üniversitesi, Fen Edebiyat Fakültesi, Kimya Bölümü, Denizli, Türkiye \\ Geliş / Received: 02/05/2018, Kabul / Accepted: 03/09/2018
}

\begin{abstract}
$\ddot{O z}$
Üstün fizikokimyasal özelliklere sahip grafen türevi nanomateryaller son yıllarda farklı araştırma alanlarında kullanılmaktadır. Bu çalışmada grafen temelli nanomateryal olan indirgenmiş grafen oksit (rGO) şeffaf iletken cam yüzeyine modifiye edilmiştir. Bu kaliteli, modifiye elektrot üzerinde sentezi başarıyla tamamlanmış ve karakterize edilmiş olan tiyenil pirol türevi elektroaktif monomer dönüşümlü voltametri yardımıyla polimerleştirilerek PSN/rGO-ITO adı verilen nanokompozit elektrot sentezlenmiştir. Burada rGO, PSN polimer yapısıyla mükemmel etkileşim gösterip, elektron transferini kolaylaştırarak kompozit filmin elektrokimyasal ve optik özelliklerini geliştirmiş̧tir. Örneğin, PSN/rGO-ITO kompoziti yalnızca PSN polimerinin kullanıldığı yüzeye göre elektrokimyasal kararlılığı döngü sayısı bakımından 5 kat artırmıştır. Yine band boşluğu enerjisi $\left(\mathrm{E}_{\mathrm{g}}\right)$ değerini $2.01 \mathrm{eV}$ 'dan $1.91 \mathrm{eV}^{\prime}$ a düşürerek iletkenliği artırmıştır. Bu kompozit film, iletken polimerlerin kullanılabileceği tüm alanlarda özellik geliştiren materyal olarak kullanılabilecek alternatif bir ürünü oluşturmaktadır.
\end{abstract}

Anahtar Kelimeler: Elektrokimya, İletken Polimer, Nanokompozit, Spektroelektrokimya

\section{Synthesis of Graphene Based Conducting-Polymer Nanocomposite Electrode and Investigation of Electrochemical and Optical Properties}

\begin{abstract}
Graphene derivative nanomaterials with superior physicochemical properties have been used in different research areas in recent years. In this work, graphene-based reduced graphene oxide (rGO) is modified to the transparent conductive glass surface. On this quality modified electrode, the nanocomposite electrode is synthesized by polymerizing (using cycling voltammetry) the thienyl pyrrole derivate electroactive monomer which has been successfully synthesized and characterized (called by PSN/rGO-ITO ). Here, the graphene based nanomaterial has excellent interaction with the polymer structure and has improved electrochemical and optical properties of composite film by facilitating electron transfer. For example, the PSN/rGO-ITO composite film has increased the electrochemical stability by 5 times in terms of the number of cycles when compared to bare PSN surface. Besides, reducing the band gap energy $\left(\mathrm{E}_{\mathrm{g}}\right)$ value from $2.01 \mathrm{eV}$ to $1.91 \mathrm{eV}$ increased the conductivity. This composite film forms an alternative product that can be used as a material for improving properties in all areas where conductive polymers can be used.
\end{abstract}

Keywords: Electrochemistry, Conducting Polymer, Nanocomposite, Spectroelectrochemistry

\section{Giriş}

Nanomateryaller üstün optik, elektriksel ve manyetik özellikleri nedeniyle, bilim ve teknolojinin çeşitli alanlarında kullanılmaya başlanmıştır. Bu tür malzemeler yüksek alana ve elektronik karakteristiklere sahip olduğu için elektroaktif moleküllerin elektron transfer reaksiyonlarını hızlandırır. Ayrıca boyutları oldukça küçük ve yüzey alanı/hacim oranları oldukça yüksektir (Abid vd., 2013). Son yıllarda, metal/ metal oksit nanopartikülleri, karbon bazlı nanomalzemeler, nano kompozitler yapıları yeni elektrot malzemelerinin hazırlanmasında kullanılmıştır. Elde edilen üstün özellikli nanokompozit malzemeler elektronik, kataliz, bilgi depolama, kimyasal algilama ve görüntüleme, çevresel iyileştirme, ilaç teslimi ve biyolojik etiketleme alanında potansiyel 
uygulamalara sahiptir (Guo ve $\mathrm{Li}, 2005$ ).

Kompozit malzeme, tek başına kullanılan özel formlarından daha iyi özelliklere sahip olan iki veya daha fazla malzemenin bir birleşimi olarak tanımlanabilir. Kompozit malzemeyi oluşturan her bir malzeme kendi fiziksel, kimyasal mekanik özelliğini korurken, oluşan kompozit malzeme yapıya katılan grupların toplam özelliklerini taşıyan daha iyi özelliklere sahip malzemedir (Campbell, 2010). Son yıllarda kompozit materyallerin kullanımında karbon temelli malzemeler tercih edilmektedir. Bu karbon temelli malzemelerin başında tek bir atomik grafit tabakası olan grafen gelmektedir. Grafen altıgen kafes haline getirilerek s1kı bir şekilde bağlanmış karbon atomlarından oluşur. Karbon atomlarının iki boyutlu altıgen bir yapıda dizilmiş bu formu, doğada iki boyutlu tek malzeme örneğini oluşturmasının yanı sıra, grafene kolay esneyebilme, birçok malzemenin yüzeyine kolay kaplanabilme gibi birçok üstün özellikler katmaktadır (Geim ve Novoselov, 2007; Han vd., 2011; Kim vd., 2011; Wang, 2011). Grafen'e oksijen atomları eklenerek elde edilen grafen oksit ise daha güçlü ve esnektir. Hidrofilik ve $\mathrm{pH}$ duyarlı olan grafen oksit düşük konsantrasyonlarda bile kompozit oluşturduğu maddenin özelliklerini artırabilmektedir. Grafene oksijen atomları eklenerek elde edilen elde edilen grafen oksitin indirgeyiciler ile reaksiyonu sonucunda hidrofobik özellikli indirgenmiş grafen oksit (rGO) elde edilmektedir (Park ve Ruoff, 2009). rGO atomik olarak daha ince olduğu için görünür spektrumda oldukça şeffaf görünümlüdür. Elektronik olarak rGO, düzensiz tek ve çok katmanlı grafenlere benzer şekilde düşük band boşluğu enerjisine sahip yarı metal gibi davranır. İndiyum kalay oksit kaplı iletken cama etkin bir alternatif platformu oluşturabilme gibi avantajlı bir özelliğe sahip olan rGO özellikle organik güneş hücreleri (Eda vd., 2008; Wu vd, 2008), organik 1şık yayan diyotlar ve ekranlar gibi cihazlarda (Dong vd., 2014; Geffroy vd., 2006; Shin vd., 2009) kullanım alanı bulmaktadır. Konjuge çift bağ içeren iletken polimerler üstün elektriksel ve optik özelliklerinden dolayı endüstride potansiyel uygulama alanına sahip fonksiyonel materyallerdir. Araştırmacıların oldukça ilgisini çeken bu malzemeler akıllı pencerelerde (Irvin ve Reynolds, 1998; Jagur-Grodzinski, 2002), organik 1şık yayan diyotlarda (OLED'ler) (Geffroy vd., 2006) ve sensörler (Ayranci vd., 2015; Bobacka ve Ivaska, 2003; Chen vd., 1999) gibi teknolojik uygulamalarda yaygin olarak kullanılmaktadır. Ayrıca teknolojideki son gelişmelere bağlı olarak yeni çok fonksiyonlu iletken polimerlerin sentezi ya da iletken polimerin modifiye edilerek özelliklerinin geliştirilmesi yönünde yapılan çalışmalar literatüre olumlu katkılar sağlamaktadır. Bu çalışmada öncelikle 3,4,5 tris(dodesiloksi)benzohidrazit kullanılarak Paal-Knorr yöntemi ile tiyenil pirol türevi elektroaktif monomer (SN) sentezlenmiş, ${ }^{1} \mathrm{H}$ NMR ile karakterize edilmiştir. İkinci aşamada ise şeffaf, iletken, indiyum kalay oksit kaplı cam (ITO cam) üzerine ticari olarak satın alınan indirgenmiş grafen oksit (rGO) basit ve hizlı olan damlat-kurut yöntemiyle immobilize edilmiş ve modifiye ITO elektrotlar hazırlanmıştır. SN monomerinin elektrokimyasal polimerizasyonunda bu modifiye elektrotlar (rGO- ITO) kullanılarak elde edilen PSN/rGO-ITO nanokompozit materyalinin elektrokimyasal karakterizasyonu dönüşümlü voltametri (CV), elektrokimyasal stabilite, artan tarama hızına karşın akım yoğunluğu artışı gibi deneylerle karakterize edilmiştir. Ayrıca spektroelektrokimyasal çalışmayla da PSN/rGO-ITO nanokompozit materyalinin optik özellikleri incelenmiştir. Elde edilen nanokompozitin yüzey karakterizasyonu FESEM cihazı ile analiz edilmiştir. Bu çalışmada ITO yüzeyine kaplanan rGO' nun iletken polimerin elektron transfer reaksiyonunu hizlandırıp, iletken polimer ile muhteşem etkileşim göstermesi ve bu etkileşimden de PSN/rGO-ITO nanokompozit malzemenin elektrokimyasal 
ve optik özelliklerinin artırılması Ticari olarak satın alınan rGO'nun 0.2

amaçlanmıştır.

\section{Materyal ve Metot}

\subsection{Kullanilan Kimyasal Maddeler ve Cihazlar}

Metil 3,4,5 tris(dodesiloksi) benzoat, lityum perklorat $\left(\mathrm{LiClO}_{4}\right)$, asetonitril Merck firmasından, tiyofen, suksinil klorür, aliminyum klorür, toluen, hidroklorik asit (HCl), dimetilformamid (DMF), sodyum bikarbonat $\left(\mathrm{NaHCO}_{3}\right)$, magnezyum sülfat $\left(\mathrm{MgSO}_{4}\right)$, p-toluen sülfonik asit (PTSA) Sigma-Aldrich firmasından temin edilmiştir. İndirgenmiş grafen oksit (rGO) SigmaAldrich firmasından satın alınmıştır. SD monomerinin ${ }^{1} \mathrm{H}-\mathrm{NMR}$ spektrumu Varian VNMRJ 400 model NMR spektrometresiyle alınmıştır. Elektrokimyasal polimerizasyon Iviumstat Potansiyostat-Galvanostat cihazı ile spektroelektrokimya deneyi ise Agilent 8453 model UV-vis spektofotometre Iviumstat Potansiyostat-Galvanostat cihazının eş zamanlı kullanımıyla gerçekleştirilmiştir. rGO immobilizasyonu ve elektrokimyasal polimerizasyonun ardından elde edilen modifiye elektrotların yüzey karakterizasyonları, Pamukkale Üniversitesi Elektron Mikroskobu Birimindeki Alan Emisyon Taramalı Elektron Mikroskobunda (FESEM-Carl Zeiss, Supra 40 VP) incelenmiştir.

\subsection{Modifiye Elektrotların Hazırlanması (rGO-ITO)}

Öncelikle modifiye edilecek ITO elektrotlar sirasıyla deterjanlı su, su ve etil alkol ile ultrasonik banyoda 10 dakika bekletilmiştir. miligramı $1 \mu$ DMF içinde iyice disperse edildikten sonra karışımın toplam hacmi DMF ile 10 ml'ye tamamlanmıştır (Ayranci vd., 2017). rGO karışımı 40 dakika ultrasonik banyoda bekletilerek disperse edilmiştir. Temizlenen ITO elektrotların belirli bölgeleri yalıtkan bantla kaplanarak aktif çalışma alanları $0.5 \quad \mathrm{~cm} * 2 \quad \mathrm{~cm}$ olacak şekilde ayarlanmıştır. $\mathrm{Bu}$ yüzeylere immobilize edilecek rGO miktarı, ITO yüzeyinde şeffaf, homojen ve çalışma yüzeyinin tamamını kaplamak koşuluyla optimize edilmiştir. Bunun için disperse edilen rGO miktarı ITO yüzeyine sirasiyla $10 \mu 1,25 \mu 1,50 \mu 1$ ve 100 $\mu 1$ olarak mikropipet yardımıyla damlatılmıştır. En şeffaf ve tüm alanı kaplayan rGO miktarı $50 \quad \mu l$ olarak belirlenmiştir. Önemli olan rGO'nun kalınlığından ziyade ITO yüzeyini en uygun şartlara getiren hacim miktarıdır. Belirlenen rGO miktarı ITO yüzeye damlatıldıktan sonra, modifiye elektrot $80^{\circ} \mathrm{C}$ de kurutulmuştur.

\subsection{SN Monomerinin Sentezi}

Öncelikle SN monomerinin, 1 (Mondal vd., 2015) ve 2 (Ayranci ve Ak, 2016) kodlu giriş maddeleri literatürde gösterildiği gibi sentezlenmiştir. 1 ( 2mmol, $1.38 \mathrm{~g}), 2$ (2 mmol, $0.5 \mathrm{~g})$ PTSA $(0.1 \mathrm{mmol}, 17 \mathrm{mg})$ varlığında $0.1 \mathrm{~mL} \quad \mathrm{DMSO} / 25 \mathrm{~mL}$ toluen içinde reaksiyona sokulmuştur. 24 saat sonunda çözücüsü uzaklaştırılan karışımdan \% 62 verimle yeşil renkli ürün elde edilmiştir (Soganci vd., 2016) (Şema 1). 1H NMR (400 $\mathrm{MHz}, \mathrm{CHCl} 3) \delta 8.65$ (s; 1H, -NH-), 7.08 (m; $8 \mathrm{H}), 6.48(\mathrm{~s} ; 2 \mathrm{H}), 3.97(\mathrm{~m} ; 6 \mathrm{H}), 1.26(\mathrm{~m}$; $60 \mathrm{H}), 0.89$ (s; 9H). 

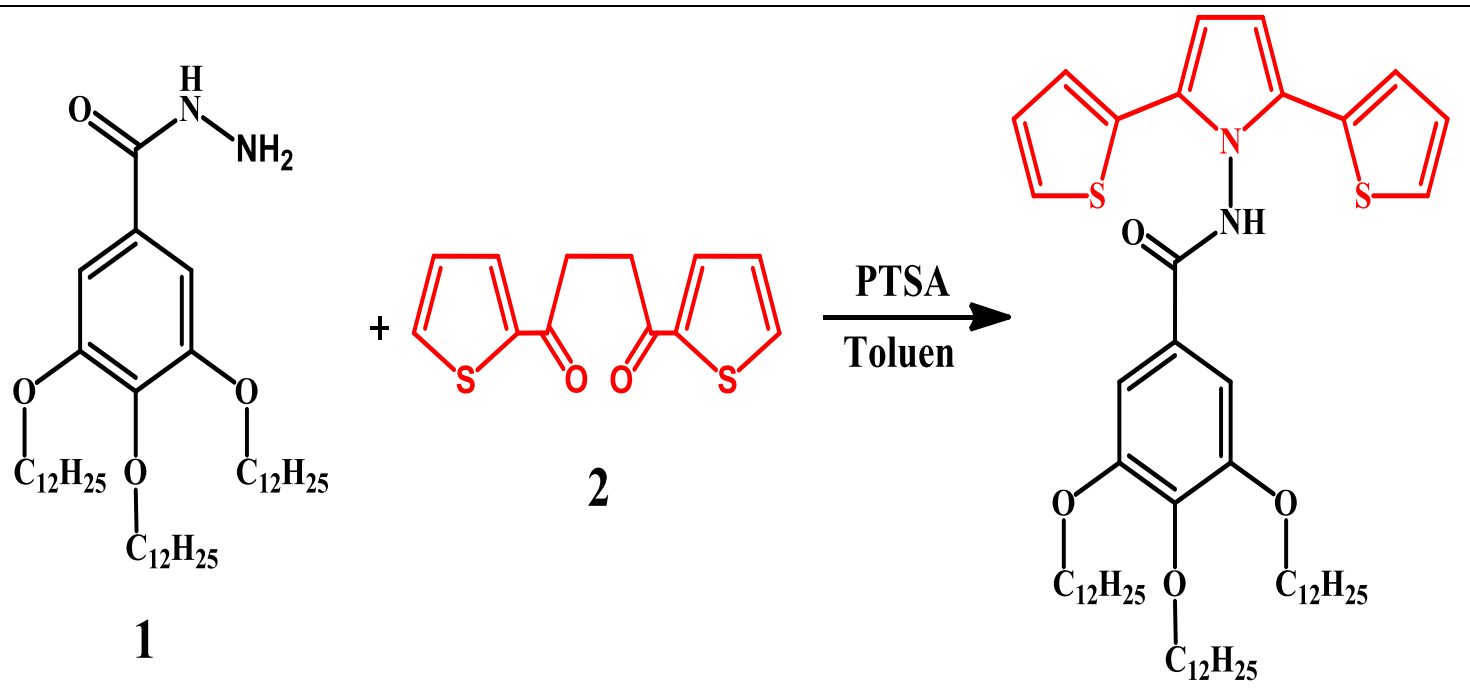

SN

$, 4,5-$

Şema 1. N-(2,5-di(tiyofen-2-il)-1H-pirol-1 tris (dodeksiloksi) benzamit (SN) sentezinin reaksiyon şeması.

\subsection{Elektrokimyasal Polimerizasyon}

Çalışma elektrodu olarak hazırlanan modifiye elektrotlar referans elektrot olarak Ag telin, karşıt elektrot olarak Pt telin kullanıldığ 3 elektrotlu elektrokimya hücresine koyulmuştur. Referans elektrot olarak kullanılan gümüş tel ferrosen $\mathrm{Fc} / \mathrm{Fc}+$ redoks çiftinin $\mathrm{E}_{1 / 2}=+0.35 \mathrm{~V}$ ' da ki potansiyeline karşıllk kalibre edilmiştir. Ivium-stat potensiyostat galvanostat cihazı yardımıyla $1 \times 10^{-3} \mathrm{M}$ SN monomeri rGO kapli modifiye elektrot üzerinde $-0.5 \quad \mathrm{~V} \quad 1.2 \mathrm{~V}$ arasında $\mathrm{LiClO}_{4} / \mathrm{ACN}$ ortamında Şekil 1' de gösterildiği gibi polimerleştirilmiştir.

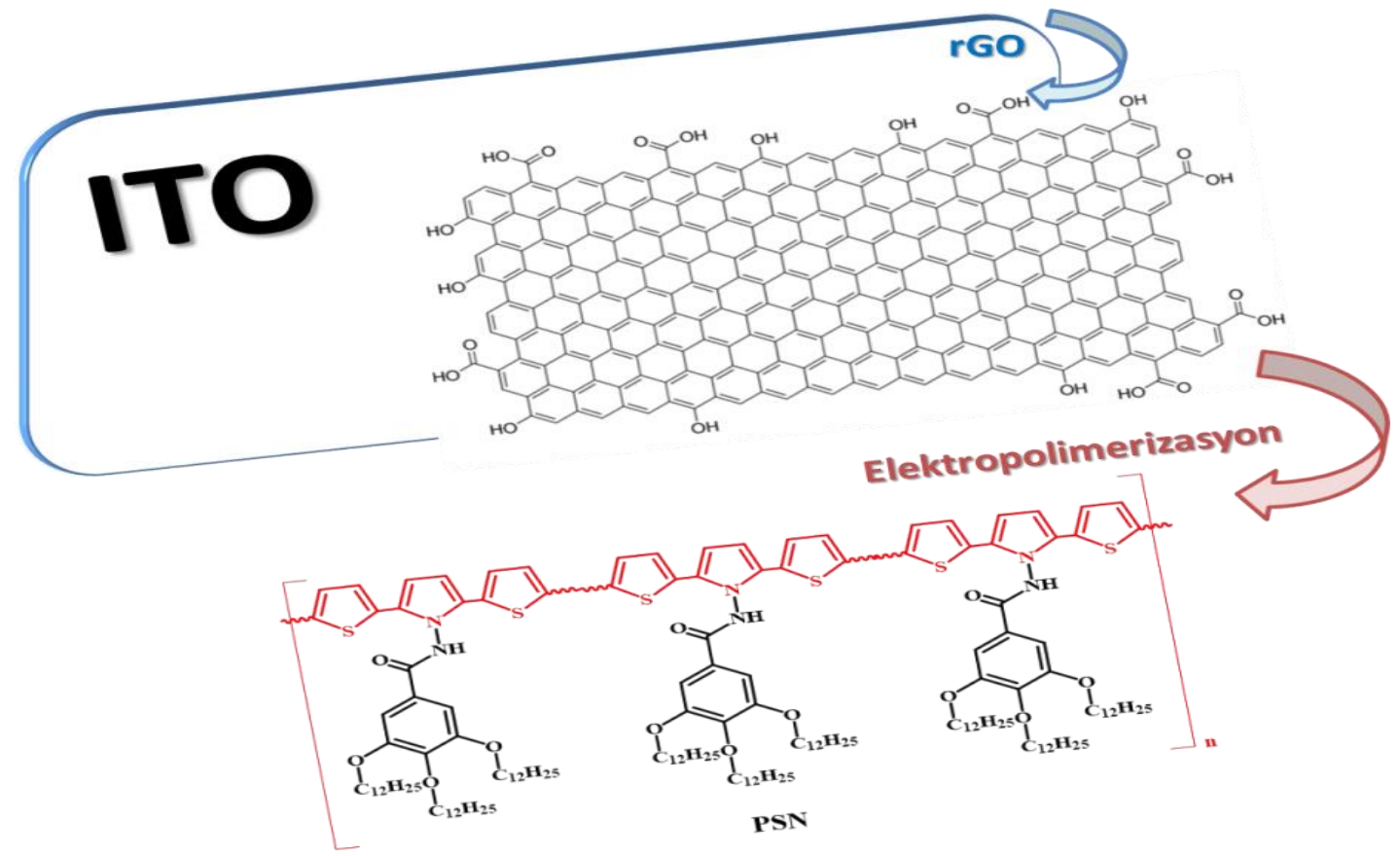

Şekil 1. PSN/rGO-ITO nanokompozit materyalinin $-0.5 \mathrm{~V} \quad 1.2 \mathrm{~V}$ arasında $\mathrm{LiClO}_{4} / \mathrm{ACN}$ ortamında elektrokimyasal polimerizasyonla hazırlanması. 


\section{Bulgular ve Tartışma}

\subsection{Modifiye Elektrodun Karakterizasyonu}

Modifiye elektrotların karakterizasyonu için ITO, rGO-ITO ve PSN/rGO-ITO elektrotları

$-0.5 \mathrm{~V} \quad 1.2 \mathrm{~V}$ arasinda $150 \mathrm{mV} / \mathrm{s}$ tarama hızıyla $\mathrm{LiClO}_{4} / \mathrm{ACN}$ çözelti ortamında dönüşümlü voltametriye (CV) tabi tutulmuştur. Şekil 2'de ki CV grafiğinden anlaşılacağı gibi rGO ITO’ya kaplandığında yük yoğunluğu artmakta, bu da PSN/rGOITO kompozitininin daha yüksek yük ve akım yoğunluğuna sahip olmasına sebep olmaktadır. Bu karakterizasyon FESEM ile de incelendiğinde görülmektedir ki, pürüzsüz görünen ITO yüzeyine rGO kaplandığında literatür ile de uyumlu olarak, yüzeyde tabaka katlanmasına sebep olan indirgenmiş grafen oksitin karakteristik yüzey görüntüsü var olmaktadir (Choi vd., 2016; Zhang ve Feng, 2010). PSN/rGO-ITO kompozitininin rGO-ITO görüntüsünden farklı olan, küresel küçük tanecikler içeren görüntüsü de kompozit oluşumunun bir kanıtıdır. rGO kendi özelliği sayesinde ITO’ya kolayca kaplanabilmiş ve PSN polimerine elektron transferini hızlandırıp kompozitin yoğun yük yoğunluğuna sebep olmasını sağlamıştır.

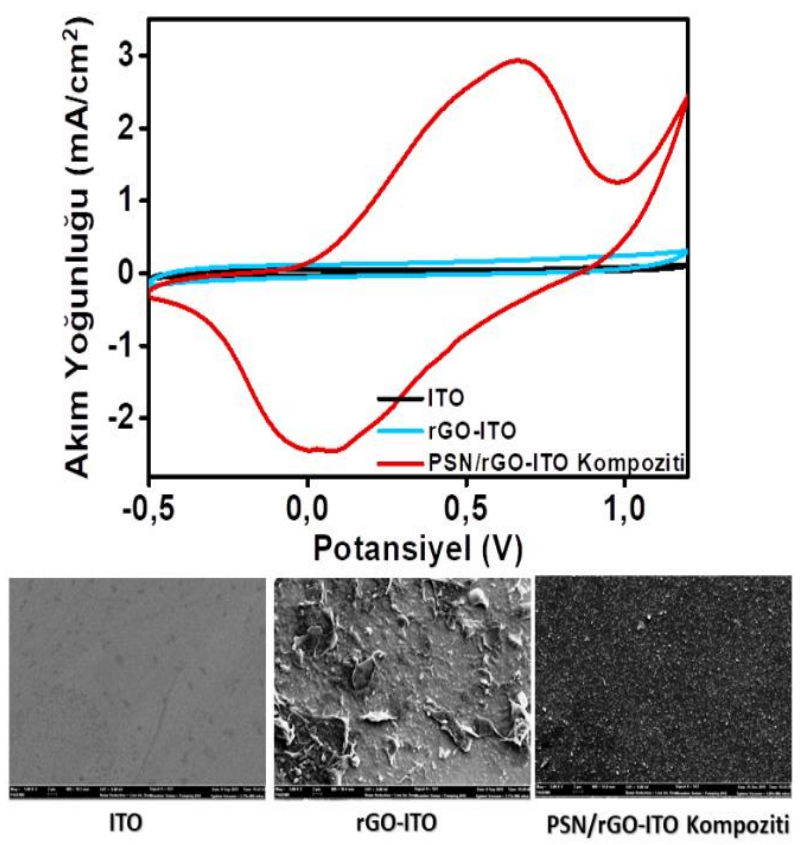

Şekil 2. ITO, rGO-ITO ve PSN/rGO-ITO elektrotlarının $-0.5 \mathrm{~V} 1.2 \mathrm{~V}$ arasında $150 \mathrm{mV} / \mathrm{s}$. tarama hızıyla $\mathrm{LiClO}_{4} / \mathrm{ACN}$ çözelti ortamındaki $\mathrm{CV}$ grafikleri ve bu şartlarda hazırlanan elektrotların FESEM mikrograf görüntüleri

\subsection{PSN/rGO-ITO Kompozitinin \\ Oluşturulması}

SN monomerinin elektrokimyasal polimerizasyonu $\quad \mathrm{LiClO}_{4} / \mathrm{ACN}$ destek elektrolit çözücü sistemi içinde -0.5 V ile 1.2 $\mathrm{V}$ arasinda $150 \mathrm{mV} / \mathrm{s}$ tarama hiziyla rGOITO çalışma elektrodu üzerinde dönüşümlü voltametri ile gerçekleştirilmiştir. Şekil 3' de gösterilen $\mathrm{CV}$ grafiği incelendiğinde, ilk döngüde SN'nin onset potansiyeli $0.47 \mathrm{~V}$ olmak üzere polimerleşme pik potansiyelleri $0.55 \mathrm{~V}$ ve $0.90 \mathrm{~V}$ olarak saptanmıştır. Bu çalışmada kompozitteki rGO, modifiye edilmemiş elektrot üzerindeki PSN polimerinin onset potansiyelini $0.57 \mathrm{~V}$ 'dan 0.47 V'a düşürerek polimerizasyonu hızlandırmış, kompozitin elektrokimyasal özelliğini artırmıştır. Modifiye elektroda kaplanan PSN polimerinin kalınlığ elektrokimyasal yolla aşağıdaki denklem yardımıyla hesaplanmıştır (Dejeu vd., 2010).

$$
\mathrm{x}: \mathrm{qM} / \rho \mathrm{AzF}
$$

Burada $\mathrm{x}$ yüzeye kaplanan polimer film kalınlığını, q polimer filmin yük yoğunluğunu, $\mathrm{M}$ monomerin mol kütlesini, $\rho$ polimerin kabul edilen yoğunluğunu, A çalışma alanını, z elektron sayısını $\mathrm{F}$ de Faraday sabitini temsil eder. q, PSN/rGOITO elektrodunun $-0.5 \mathrm{~V} \quad 1.2 \mathrm{~V}$ arasında $\mathrm{LiClO}_{4} / \mathrm{ACN}$ ortamında alınan $\mathrm{CV}$ grafiğinin alanından hesaplanan yük yoğunluğu miktarı $3.48 \mathrm{mCcm}^{-2}$ olarak hesaplanmıştır. Çalışma alanı $1 \mathrm{~cm}^{2}$ 'dir. Diğer sabitler yerine koyularak modifiye elektrotta kaplanan PSN polimerinin kalınlığı $0.09 \mu \mathrm{m}$ olarak hesaplanmıştır. 


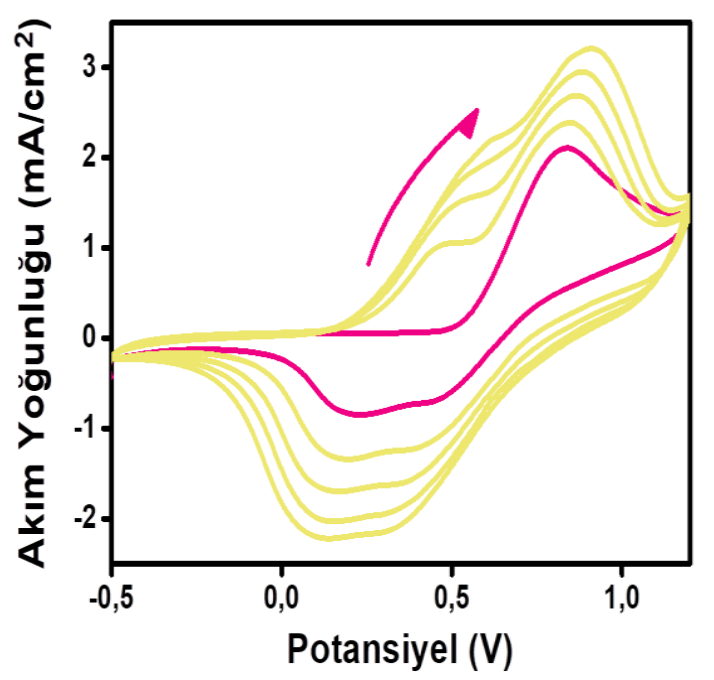

Şekil 3. PSN/rGO-ITO kompozitinin $\mathrm{LiClO}_{4} / \mathrm{ACN}$ destek elektrolit çözücü sistemi içinde $-0.5 \mathrm{~V}$ ile $1.2 \mathrm{~V}$ arasında $150 \mathrm{mV} / \mathrm{s}$ tarama hızıyla alınan dönüşümlü voltametri grafiği.

\subsection{PSN/rGO-ITO Kompozitinin Elektrokimyasal Karakterizasyonu}

Hazırlanan PSN/rGO-ITO kompozitinin monomersiz $\mathrm{LiClO}_{4} / \mathrm{ACN}$ çözücü sistemine alınmıştır. $\mathrm{Bu}$ sistemde PSN/rGO-ITO kompozitinin farklı tarama hızlarındaki akım yoğunluğu değişimini incelemek amaciyla, $50 \mathrm{mV} / \mathrm{s}$ 'den $500 \mathrm{mV} / \mathrm{s}$ 'ye kadar farklı tarama hızları uygulaması yapılmıştır. Tarama hızı arttıkça akım yoğunluğu ve yük yoğunluğu artmıştır (Şekil 4).

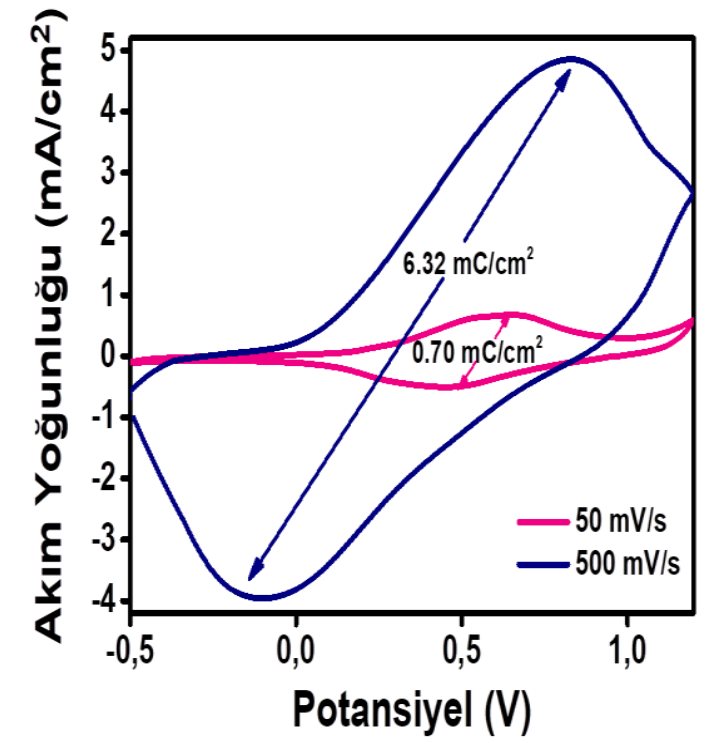

Şekil 4. PSN/rGO-ITO kompozitinin farklı tarama hızlarında monomersiz $\mathrm{LiClO}_{4} / \mathrm{ACN}$ ortamında dönüşümlü voltametri grafiklerinin karşılaştırılması.
Dönüşümlü voltametri iletken polimerlerin uzun vadeli elektrokimyasal kararlılığının ölçümünde kullanılabilmektedir. PSN/rGOITO kompozitinin kararlılığını test etmek için, elektrokimyasal polimerizasyonla hazırlanan kompozit film, monomersiz çözücü sisteminde $-0.5 \mathrm{~V} 1.2 \mathrm{~V}$ arasında 500 $\mathrm{mV} / \mathrm{sn}$ 'lik bir tarama hızı ile 1000 döngü dönüşümlü voltametriye maruz birakılmıştır. Şekil 5' de gözlendiği gibi 1000. döngü sonras1 PSN/rGO-ITO kompozit filmde yüklenen yük miktarı ilk döngüye göre \% 98'ini koruyabilmiştir. Modifiye edilmemiş elektrot üzerindeki PSN polimer filmi elektrokimyasal stabilitesini 200 döngü boyunca koruyabiliyorken, PSN/rGO-ITO kompoziti 1000 döngü boyunca kararlılık göstermiştir. $\mathrm{Bu}$ da rGO'nun polimer yüzeyiyle harika bir etkileşim gösterdiğini polimerin yüzey alanı aktifliğini 5 kat fazla koruduğunu kanıtlamaktadır.

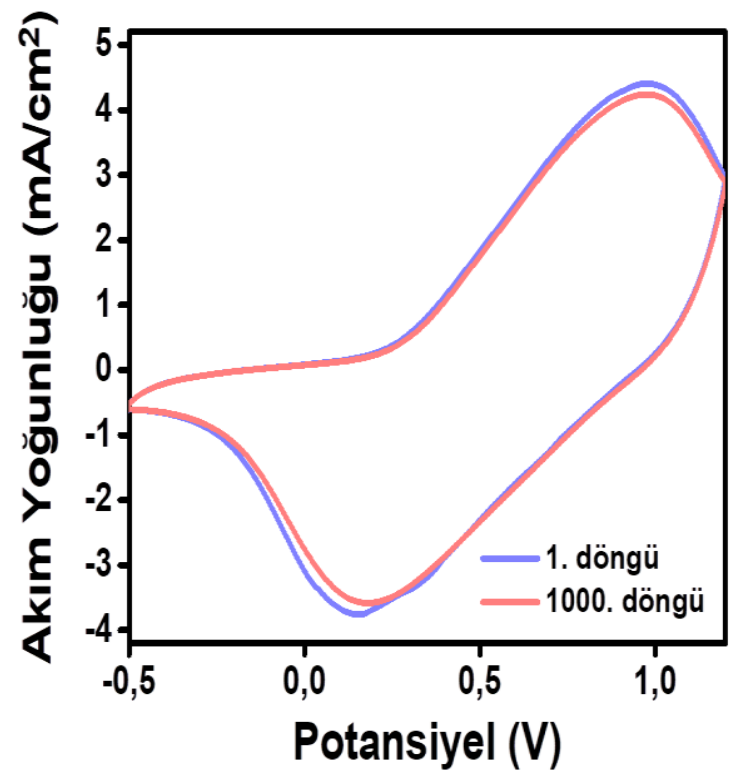

Şekil 5. PSN/rGO-ITO kompozitinin $500 \mathrm{mV} / \mathrm{s}$ tarama hızında monomersiz $\mathrm{LiClO}_{4} / \mathrm{ACN}$ ortamında 1000 döngü boyunca gösterdiği elektrokimyasal kararlılık.

\subsection{PSN/rGO-ITO Kompozitinin Spektroelektrokimyasal Özellikleri}

Elektrokimyasal polimerizasyonu $-0.5 \mathrm{~V}$ ile $1.2 \mathrm{~V}$ arasında 10 döngü dönüşümlü voltametri ile $\mathrm{LiClO}_{4} / \mathrm{ACN}$ çözücü sisteminde $150 \mathrm{mV} / \mathrm{s}$ tarama hızıyla rGO- 
ITO elektrodu üzerinde gerçekleştirilen Yapılan çalı̧̧ada tercih edilen malzemenin PSN/rGO-ITO kompoziti monomersiz spektrolektrokromik özellikleri önemli çözücü sistemine alınmıştır. Kompozitin ölçüde geliştirmesinin literatür ile uyumlu indirgenme ve yükseltgenme potansiyelleri arasında UV-spektrumları alınmış, $\pi-\pi^{*}$ geçişlerine ait maksimum dalga boyları $\left(\lambda_{\max }\right) \quad 458 \mathrm{~nm}$ olarak ölçülmüştür. $\pi-\pi^{*}$ geçişlerine ait bant boşluğu enerjisi $\left(\mathrm{E}_{\mathrm{g}}\right.$ değeri) $1.91 \mathrm{eV}$ olarak ölçülmüştür. -0.5 V'dan 1.2 V'a kadar uygulanan potansiyeller arttıça, $455 \mathrm{~nm}$ ' de ki $\pi-\pi^{*}$ geçişlerine ait absorpsiyon piki azalmış $909 \mathrm{~nm}$ 'de polaron bandları gözlenmiştir (Şekil 6a). Polimer filmi nötral halden yükseltgenmiş hale geçerken açık sarı, yeşil ve koyu mavi renklerini göstermiştir (Şekil 6b). PSN/rGOITO kompozit filminin $458 \mathrm{~nm}$ ve $909 \mathrm{~nm}$ 'de gösterdiği geçirgenlik oranı sırasıyla $\% 33$ ve \%54 olarak ölçülmüştür. Bu sonuç rGO'nun hızlı elektron transferi sağlayıp (Saxena vd., 2011) (Tang Longhua, vd., 2009) PSN polimeriyle çok iyi etkileşim gösterdiğini ve bu etkileşimin polimerin indirgenmiş ve yükseltgenmiş halleri arasındaki optiksel zıtlığını artırdığını kanıtlamaktadır. Literatüre bakıldığında karbon temelli nanomalzemeler içinde özellikle şeffaf elektronik materyallerde $\mathrm{rGO}^{\prime}$ nun görünür spektrumda daha şeffaf özellik göstermesi sebebiyle tercih sebebi olduğu görülmektedir.

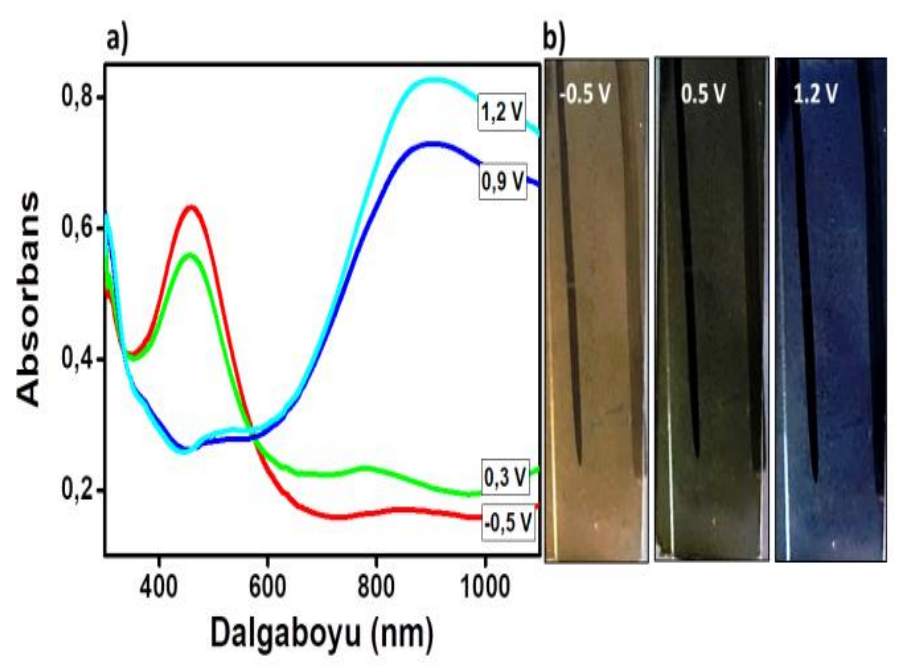

Şekil 6. a) PSN/rGO-ITO kompozitinin monomersiz $\mathrm{LiClO}_{4} / \mathrm{ACN}$ ortamında farklı dalgaboylarındaki a) absorbans değişimi b) redoks renkleri.

PSN/rGO-ITO kompozitinin elektrokimyasal özellikleri modifiye edilmemiş elektrot üzerindeki PSN polimer filmi ile karşılaştırılıp sonuçlar Tablo 1' de özetlenmiş̧tir. Buradan rGO' nun polimer yapısına geçen elektron transfer reaksiyonunu hızlandırdığı ve polimer ile kuvvetli bir şekilde etkileştiği gözlenmektedir.

Tablo 1. PSN/rGO-ITO kompozitinin modifiye edilmemiş elektrot üzerindeki PSN polimer filmi ile karşılaştırılması.

\begin{tabular}{|c|c|c|c|c|c|}
\hline Malzeme & $\begin{array}{l}\text { Elektrokimyasal } \\
\text { Kararlılık }\end{array}$ & $\begin{array}{l}\lambda \max \\
(\mathbf{n m})\end{array}$ & $\% \Delta T$ & $\begin{array}{l}\mathrm{Eg} \\
(\mathrm{eV})\end{array}$ & Referans \\
\hline PSN/rGO-ITO & $\% 99 / 1000$ döngü & $\begin{array}{l}458 \\
909\end{array}$ & $\begin{array}{l}33(458 \mathrm{~nm} \text { için }) \\
54 \text { (909 } \mathrm{nm} \text { için) }\end{array}$ & 1,91 & $\mathrm{Bu}$ çalışma \\
\hline P(TPDOB) & $\% 99 / 200$ döngü & $\begin{array}{l}455 \\
835\end{array}$ & $\begin{array}{l}22(455 \mathrm{~nm} \text { için }) \\
47 \text { (835 } \mathrm{nm} \text { için })\end{array}$ & 2,01 & $\begin{array}{l}\text { (Soganci et al., } \\
2016 \text { ) }\end{array}$ \\
\hline
\end{tabular}

\section{Sonuç ve Tartışma}

rGO ile modifiye edilmiş ITO yüzeyinde elektrokimyasal polimerizasyonla hazırlanan PSN/rGO-ITO kompozit filminin elektrokimyasal ve spektroelektrokimyasal özellikleri başarıyla incelenmiştir. PSN/rGOITO kompozit filminin modifiye edilmemiş elektrot üzerindeki PSN polimer filminin elektrokimyasal kararlılığından dönüşümlü voltametri döngü sayısı bakımından 5 kat daha fazla olduğu kanıtlanmıştır. Yine rGO polimer yüzeyiyle mükemmel etkileşerek polimerin indirgenme yükseltgenme piklerini değiştirmiş ve PSN/rGO-ITO kompozit filminin optiksel zitlığını düşük dalgaboyunda \%22' den \%33'e yüksek 
dalgaboyunda \%47'den \%54'e artırmıştır.

Özetle PSN/rGO-ITO kompozit filmi elektrokromik ve elektronik cihaz tasarımları için sinerjik etki ile özellik geliştirebilecek alternatif bir platform oluşturmuştur.

\section{Kaynaklar}

Abid, A.D., Anderson, D.S., Das, G.K., Van Winkle, L.S., Kennedy, I.M. 2013. Novel lanthanide-labeled metal oxide nanoparticles improve the measurement of in vivo clearance and translocation. Particle and Fibre Toxicology, 10, 1. doi:10.1186/17438977-10-1.

Ayranci, R., Ak, M. 2016. Synthesis of a novel, fluorescent, electroactive and metal ion sensitive thienylpyrrole derivate. New Journal Chemistry, 40, 8053-8059. doi:10.1039/c6nj02006b.

Ayranci, R., Baskaya, G., Guzel, M., Bozkurt, S., Ak, M., Savk, A., Sen, F. 2017. Enhanced optical and electrical properties of PEDOT via nanostructured carbon materials: A comparative investigation. NanoStructures \& Nano-Objects 11, 13-19. doi:10.1016/j.nanoso.2017.05.008.

Ayranci, R., Soganci, T., Guzel, M., Demirkol, D.O., Ak, M., Timur, S. 2015. Comparative investigation of spectroelectrochemical and biosensor application of two isomeric thienylpyrrole derivatives. RSC Advances. 5, 52543-52549. doi:10.1039/C5RA07247F.

Bobacka, J., Ivaska, A. 2003. Chemical Sensors Based on Conducting Polymers. Materials and Applications. 15 , 173-187. doi:10.1002/9783527630592.ch9.

Campbell, F.C. 2010. Product Performance Evulation with CAD/CAE. Structural Analysis. 43-119. doi:10.1016/B978-012-398460-9.00002-0.

Chen, L., McBranch, D.W., Wang, H.L., Helgeson, R., Wudl, F., Whitten, D.G. 1999. Highly sensitive biological and chemical sensors based on reversible fluorescence quenching in a conjugated polymer. Proceedings of the National
Academy of Sciences of the United States of America, 96, 12287-12292. doi:10.1073/pnas.96.22.12287.

Choi, Y.J., Kim, E., Han, J.W., Kim, J.H., Gurunathan, S. 2016. A novel biomolecule-mediated reduction of graphene oxide: A multifunctional anticancer agent. Molecules 21. doi:10.3390/molecules21030375.

Dejeu, J., Taouil, A.E., Rougeot, P., Lakard, S., Lallemand, F., Lakard, B. 2010. Morphological and adhesive properties of polypyrrole films synthesized by sonoelectrochemical technique. Synthetic Metals. 160, 2540-2545. doi:10.1016/j.synthmet.2010.10.002.

Dong, P., Zhu, Y., Zhang, J., Peng, C., Yan, Z., Li, L., Peng, Z., Ruan, G., Xiao, W., Lin, H., Tour, J.M., Lou, J. 2014. Graphene on Metal Grids as the Transparent Conductive Material for Dye Sensitized Solar Cell. The Journal of Physical Chemistry C. 118, 2586325868. doi:10.1021/jp505735j.

Eda, G., Fanchini, G., Chhowalla, M. 2008. Large-area ultrathin films of reduced graphene oxide as a transparent and flexible electronic material. Nature Nanotechnology 3, 270-274. doi:10.1038/nnano.2008.83.

Eda, G., Lin, Y.Y., Miller, S., Chen, C.W., Su, W.F., Chhowalla, M. 2008b. Transparent and conducting electrodes for organic electronics from reduced graphene oxide. Applied Physics Letters. 92, 233-305. doi:10.1063/1.2937846.

Geffroy, B., le Roy, P., Prat, C. 2006. Organic light-emitting diode (OLED) technology: Materials, devices and display technologies. Polymer International 55, 572-582. doi:10.1002/pi.1974.

Geim, A.K., Novoselov, K.S. 2007. The rise of graphene. Nature Materials. 6, 183191. doi:10.1038/nmat1849.

Guo, D.J., Li, H.L. 2005. Highly dispersed Ag nanoparticles on functional MWNT surfaces for methanol oxidation in alkaline solution. Carbon, 43, 12591264.doi:10.1016/j.carbon.2004.12.021 
Han, P., Yue, Y., Liu, Z., Xu, W., Zhang, L., $\mathrm{Xu}$, H., Dong, S., Cui, G., Sum, E., Rychcik, M., Skyllas-kazacos, M., Sum, E., Skyllas-Kazacos, M., SkyllasKazacos, M., Rychcik, M., Robins, R.G., Fane, A.G., Green, M.A., Yue, L., Li, W.S., Sun, F.Q., Zhao, L.Z., Xing, L.D., Chen, D.Y., Wang, S.J., Xiao, M., Meng, Y.Z., Li, X.F., Zhang, H.M., Mai, Z.S., Zhang, H.Z., Vankelecom, I., Zhang, H.Z., Zhang, H.M., Li, X.F., Mai, Z.S., Zhang, J.H., Gattrell, M., Qian, J., Stewart, C., Graham, P., MacDougall, B., Gattrell, M., Park, J., MacDougall, B., Apte, J., McCarthy, S., Wu, C.W., Zhong, S., Padeste, C., Kazacos, M., SkyllasKazacos, M., Kaneko, H., Nozaki, K., Wada, Y., Aoki, T., Negishi, A., Kamimoto, M., Sun, B., SkyllasKazakos, M., Fabjan, C., Garche, J., Harrer, B., Jörissen, L., Kolbeck, C., Philippi, F., Tomazic, G., Wagner, F., Dikin, D.A., Stankovich, S., Zimney, E.J., Piner, R.D., Dommett, G.H.B., Evmenenko, G., Nguyen, S.T., Ruoff, R.S., Kim, F., Cote, L.J., Huang, J.X., Zhou, X.F., Liu, Z.P., Wassei, J.K., Cha, K.C., Tung, V.C., Yang, Y., Kaner, R.B., Becerril, H.A., Mao, J., Liu, Z.F., Stoltenberg, R.M., Bao, Z.N., Chen, Y.S., Xu, Y.X., Sheng, K.X., Li, C., Shi, G.Q., Dong, X.C., Su, C.Y., Zhang, W.J., Zhao, J.W., Ling, Q.D., Huang, W., Chen, P., Li, L.J., Pumera, M., Sun, Y.Q., Wu, Q., Shi, G.Q., Aboutalebi, S.H., Chidembo, A.T., Salari, M., Konstantinov, K., Wexler, D., Liuac, H.K., Doua, S.X., Han, P.X., Wang, H.B., Liu, Z.H., Chen, X., Ma, W., Yao, J.H., Zhu, Y.W., Cui, G.L., Hummers, W.S., Offeman, R.E., Xu, Y.X., Bai, H., Lu, G.W., Li, C., Shi, G.Q., Chen, H.Y., Wang, N., Di, J.C., Zhao, Y., Song, Y.L., Jiang, L., Geng, Y., Wang, S.J., Kim, J.K., Tang, Y., Gou, J.H., Byon, H.R., Lee, S.W., Chen, S., Hammond, P.T., Shao-Horn, Y., Kim, Y.K., Min, D.H., Cai, D.Y., Song, M., Xu, C.X., Liu, J.C., Bai, H.W., Wang, Y.J., Liu, Z.Y., Zhang, X.W., Sun, D.D., Shinde, D.B., Debgupta, J., Kushwaha, A., Aslam, M., Pillai, V.K., Ovejero, G., Sotelo, J.L., Romero, M.D., Rodríguez, A.,
Ocaña, M.A., Rodríguez, G., García, J., Shen, J.F., Li, N., Shi, M., Hu, Y.Z., Ye, M.X., Kudin, K.N., Ozbas, B., Schniepp, H.C., Prud'homme, R.K., Aksay, I.A., Car, R., Shen, J.F., Hu, Y.Z., Shi, M., Lu, X., Qin, C., Li, C., Ye, M.X., Sahoo, N.G., Cheng, H.K.F., Li, L., Chan, S.H., Judeh, Z., Zhao, J.H., Rao, C.N.R., Biswas, K., Subrahmanyama, K.S., Govindaraj, A., Seredych, M., Tamashausky, A. V., Bandosz, T.J., Mattevi, C., Eda, G., Agnoli, S., Miller, S., Mkhoyan, K.A., Celik, O., Mastrogiovanni, D., Granozzi, G., Garfunkel, E., Chhowalla, M., Xu, Y.J., Liu, X., Cui, G.L., Zhu, B., Weinberg, G., Schlögl, R., Maier, J., Su, D.S., Huang, H., Liu, X.M., Zhang, X.Y., Liu, W.L., Su, X.G., Zhang, Z.Q., Haddadi-Asl, V., Kazacos, M., Skyllas-Kazacos, M., Xue, F.Q., Wang, Y.L., Wang, W.H., Wang, X.D., Sun, B., Skyllas-Kazacos, M., Sun, B., Skyllas-Kazacos, M., Lv, R.T., Cui, T.X., Jun, M.S., Zhang, Q., Cao, A.Y., Su, D.S., Zhang, Z.J., Yoon, S.H., Miyawaki, J., Mochida, I., Kang, F.Y., Wang, W.H., Wang, X.D., Meyers, J.P., Doyle, M., Darling, R.M., Newman, J., Albery, W.J., Mount, A.R., Xiao, P., Gao, W.Y., Qiu, X.P., Zhu, W.T., Sun, J., Chen, L.Q., Yang, S.B., Feng, X.L., Zhi, L.J., Cao, Q., Maier, J., Müllen, K. 2011. Graphene oxide nanosheets/multi-walled carbon nanotubes hybrid as an excellent electrocatalytic material towards $\mathrm{VO} 2+/ \mathrm{VO} 2+$ redox couples for vanadium redox flow batteries. Energy \& Environmental Science 4, 4710. doi:10.1039/c1ee01776d.

Irvin, D.J., Reynolds, J.R. 1998. Tuning the band gap of easily oxidized bis(2thienyl)- and bis(2-(3,4ethylenedioxythiophene))-phenylene polymers. Polymers for Advanced Technologies. 9, 260-265.

Jagur-Grodzinski, J. 2002. Electronically conductive polymers. Polymers for Advanced Technologies 13, 615-625. doi:10.1002/pat.285.

Kim, H., Kobayashi, S., AbdurRahim, M.A., Zhang, M.J., Khusainova, A., Hillmyer, 
M.A., Abdala, A.A., Macosko, C.W. 2011. Graphene/polyethylene nanocomposites: effect of polyethylene functionalization and blending methods. Polymer, 52, 1837-1846.

Mondal, S., Chakraborty, P., Bairi, P., Chatterjee, D.P., Nandi, A.K. 2015. Light induced $\mathrm{E}-\mathrm{Z}$ isomerization in a multi-responsive organogel: elucidation from 1 H NMR spectroscopy. Chemical Communications. 51, 10680-10683. doi:10.1039/C5CC03609G.

Park, S., Ruoff, R.S. 2009. Chemical methods for the production of graphenes. Nature Nanotechnolgy. 4, 217-224. doi:10.1038/nnano.2009.58.

Saxena, A.P., Deepa, M., Joshi, A.G., Bhandari, S., Srivastava, A.K. 2011. Poly (3 , 4-ethylenedioxythiophene) Ionic Liquid Functionalized Graphene / Reduced Graphene Oxide Nanostructures: Improved Conduction and Electrochromism. Applied Materials and Interfaces. 3(4), 11151126. doi: 10.1021/am101255a.

Shin, H.J., Kim, K.K., Benayad, A., Yoon, S.M., Park, H.K., Jung, I.S., Jin, M.H., Jeong, H.K., Kim, J.M., Choi, J.Y., Lee, Y.H. 2009. Efficient reduction of graphite oxide by sodium borohydride and its effect on electrical conductance. Advanced Functional Materials 19, 1987-1992.

doi:10.1002/adfm.200900167.

Soganci, T., Soyleyici, H.C., Ak, M. 2016. A soluble and fluorescent new type thienylpyrrole based conjugated polymer: optical, electrical and electrochemical properties. Physical Chemistry Chemical Physics. 18, 14401-14407. doi:10.1039/C6CP02214F.

Tang, L., Wang, Y., Li, Y., Feng, H., Lu, J., Li, J. 2009. Preparation, structure, and electrochemical properties of reduced graphene sheet films. Advanced Functional Materials. 19, 2782-2789. doi:10.1002/adfm.200900377.
Wang, J. 2011. "Graphene in Electroanalysis." Electroanalysis 23, 801. doi:10.1002/elan.201190006.

Wu, J., Becerril, H.A., Bao, Z., Liu, Z., Chen, Y., Peumans, P. 2008. Organic solar cells with solution-processed graphene transparent electrodes. Applied Physics Letters. 92. doi:10.1063/1.2924771.

Zhang, H., Feng, P.X. 2010. Fabrication and characterization of few-layer graphene. Carbon 48, 359-364. doi:10.1016/j.carbon.2009.09.037. 